\title{
Thymosin $\beta 4$ reduces senescence of endothelial progenitor cells via the PI3K/Akt/eNOS signal transduction pathway
}

\author{
JUAN LI, FUYU QIU, LU YU, YANBO ZHAO, GUOSHENG FU and BINQUAN ZHOU \\ Department of Cardiology, Biomedical Research (Therapy) Center, Sir Run Run Shaw Hospital, \\ College of Medicine, Zhejiang University, Hangzhou 310016, Zhejiang, P.R. China
}

Received August 4, 2012; Accepted October 30, 2012

DOI: $10.3892 / \mathrm{mmr} .2012 .1180$

\begin{abstract}
We previously demonstrated that thymosin $\beta 4$ (T $\beta 4$ ) regulates a variety of endothelial progenitor cell (EPC) functions, including cell migration, proliferation, survival and angiogenesis. However, the effect of T $\beta 4$ on the senescence of circulating EPCs remains unclear. In the present study, the effect of T $\beta 4$ on EPC senescence and the signal transduction pathways involved in this process was investigated. Circulating EPCs isolated from healthy volunteers were cultured in the absence or presence of $\mathrm{T} \beta 4$ and various signal cascade inhibitors. T $\beta 4$ inhibited EPC senescence in a concentration-dependent manner. In addition, $\mathrm{T} \beta 4$ increased telomerase activity and expression of telomerase reverse transcriptase mRNA in EPCs. T $\beta 4$ also regulated the expression of p21, p27 and cyclin D1. The effects of T $\beta 4$ on EPC senescence were eliminated by the phosphoinositide 3'-kinase (PI3K) inhibitor, wortmannin and the endothelial nitric oxide synthase inhibitor, L-nitroarginine methyl ester hydrochloride (L-NAME). In conclusion, the inhibitory effect on EPC senescence mediated by $\mathrm{T} \beta 4$ may be attributed, at least in part, to activation of the PI3K-Akt-eNOS signaling pathway.
\end{abstract}

\section{Introduction}

Endothelial progenitor cells (EPCs) are a specific subtype of hematopoietic stem cells present in peripheral blood that express various combinations of antigens traditionally associated with hematopoietic stem and endothelial cells, including kinase insert domain-containing receptor, CD34 and CD133 (1). Previous studies have identified that EPCs contribute to neovascularization of ischemic tissue and reversal of endothelial dysfunction $(2,3)$, indicating a poten-

Correspondence to: Dr Binquan Zhou, Department of Cardiology, Biomedical Research (Therapy) Center, Sir Run Run Shaw Hospital, College of Medicine, Zhejiang University, 3 East Qingchun Road, Hangzhou 310016, Zhejiang, P.R. China

E-mail: benzhou@hotmail.com

Key words: thymosin $\beta 4$, endothelial progenitor cell, cell senescence tial application in cell therapy to augment vascularization in patients with ischemic heart disease (4). However, there are limited numbers of EPCs in the circulating blood $(<0.05 \%$ of leukocytes) (5) and the number and functional activity of EPCs in healthy volunteers and patients with coronary artery disease is affected by various risk factors, including aging, diabetes, hypercholesterolemia, hypertension and smoking (6). Allogeneic transfusion of EPCs from healthy donors leads to immunological incompatibility in specific individuals; therefore, the functional enhancement of autologous EPCs, including enhanced mobilization, homing, survival and secretion of growth factors in a paracrine or autocrine manner, must be developed for EPC-based therapies for cardiovascular diseases.

Our previous studies demonstrated that thymosin $\beta 4$ (T $\beta 4$ ) increases EPC migration and decreases EPC apoptosis under serum deprivation via the phosphoinositide 3'-kinase (PI3K)-Akt-endothelial nitric oxide synthase (eNOS) signal transduction pathway $(7,8)$. However, the effect of $\mathrm{T} \beta 4$ on senescence of circulating EPCs remains unresolved. Cellular aging or senescence is characterized by cell cycle arrest and is triggered by various signaling pathways (9). Understanding of the mechanisms underlying cellular senescence has developed significantly, and senescence is currently associated with attrition (i.e., reduced length) of telomeres (10). Telomerase, a ribonucleoprotein with reverse transcriptase activity, extends telomeres of eukaryotic chromosomes and delays the development of senescence (11). Furthermore, a senescent phenotype is induced by expression of cyclin-dependent kinase inhibitors (CDKIs) (12). The cyclin-dependent kinases (CDKs) are central to the coordination of the eukaryotic cell cycle and function to integrate diverse growth regulatory signals. The majority of anti-proliferative signals lead to induction of CDKIs, including $\mathrm{p} 27^{\mathrm{Kip} 1}$ and $\mathrm{p} 21^{\mathrm{Cip} 1 / \text { Waf1 }}$ and therefore these cell cycle inhibitors have been studied as biomarkers of senescence (12).

The aim of the present study was to investigate the effects of T $\beta 4$ on EPC senescence and telomerase activity and to define the signal transduction pathway involved in this process.

\section{Materials and methods}

Isolation, cultivation and characterization of circulating EPCs. EPCs were isolated, cultured and characterized according to previously described techniques $(13,14)$. Briefly, 
peripheral blood mononuclear cells were isolated from healthy volunteers by density-gradient centrifugation with Ficoll (Cedarlane Laboratories Ltd., Hornby, ON, Canada) according to the manufacturer's instructions. All blood samples were obtained, processed and analyzed individually in independent experiments. Following purification with 3 washing steps, $1 \times 10^{7}$ peripheral blood mononuclear cells were plated on fibronectin-coated 6-well plates (Chemicon, Temecula, CA, USA). Cells were cultured in endothelial basal medium-2 (Clonetics Corp., Walkersville, MD, USA) with single aliquots of EGM-2MV containing 5\% fetal bovine serum, vascular endothelial growth factor, fibroblast growth factor-2, epidermal growth factor, insulin-like growth factor and ascorbic acid. Following 4 days in culture, non-adherent cells were removed by washing with phosphate-buffered saline (PBS), fresh medium was applied and the culture was maintained for 7 days. The study was approved by the ethics committee of Zhejiang University, and performed in accordance with the 1964 Declaration of Helsinki. Informed consent was obtained from all volunteers.

Senescence-associated $\beta$-galactosidase (SA- $\beta$-gal) staining. Following incubation for 7 days, the culture medium was replaced with fresh medium and cells were cultured in the absence or presence of $\mathrm{T} \beta 4(0,1,10,100$ and 1,000 ng/ml) (ProSpec-Tany TechnoGene Ltd., Rehovot, Israel) for a further $24 \mathrm{~h}$. EPCs were harvested and SA- $\beta$-gal activity was measured with the Senescence $\beta$-gal Staining kit (Cell Signaling Technology Inc., Danvers, MA, USA) as described previously (15).

Real-time reverse transcription-polymerase chain reaction (RT-PCR). Total RNA was extracted from EPCs with TRIzol (Invitrogen Life Technologies, Carlsbad, CA, USA) according to a modification of the method described by Chirgwin et al (16). RNA (1-2 $\mu \mathrm{g})$ was converted into cDNA using murine leukemia virus reverse transcriptase (Promega Corp., Madison, WI, USA). Transcribed cDNA was then used as a template in PCR amplification to measure the expression of hTERT. Primers used for hTERT PCR were as follows: sense, 5'-AGA GTG TCT GGA GCA AGT TGC-3'; and antisense, 5'-CGT AGT CCA TGT TCA CAA TCG-3'. Amplification produced a 182-bp hTERT fragment (nucleotides 1789-1971; GenBank no. AF018167) that was verified by southern blot analysis and nested PCR as previously described (17). Primers used for GAPDH PCR were as follows: sense, 5'-GGG TGT GAA CCA TGA GAA GT-3'; and antisense, 5'-GAC TGT GGT CAT GAG TCC T-3'. GAPDH was amplified as a reference and produced a 136-bp product. Real-time PCR was performed using an ABI 7500 cycler (Applied Biosystems, Foster City, CA, USA) with SYBR-Green PCR mix (Takara, Shiga, Japan) according to the manufacturer's instructions. These experiments were repeated 3 times for each cell line.

Detection of telomerase activity. EPCs were washed in PBS, lysed with $30 \mu 1$ lysis buffer and the telomeric repeat amplification protocol (TRAP) assay for telomerase activity was performed using the TeloTAGGG Telomerase PCR ELISA Plus Kit (Roche Diagnostics, Indianapolis, IN, USA) according to the manufacturer's instructions, as described previously (18).
A
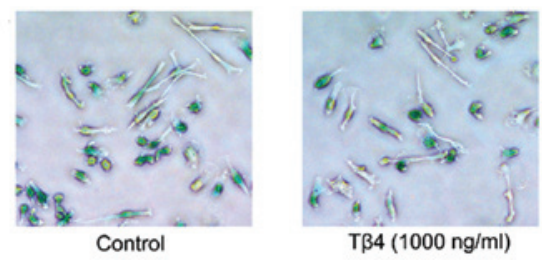

B

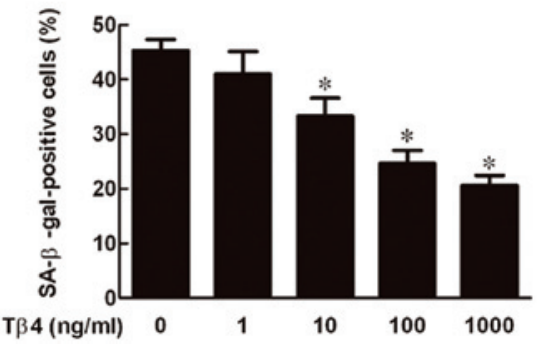

Figure 1. Effect of T $\beta 4$ on EPC senescence as assessed by acidic-galactosidase activity staining. (A) Representative photomicrographs reveal SA- $\beta$-gal-positive cells (blue) in an EPC subpopulation treated with or without $1,000 \mathrm{ng} / \mathrm{ml} \mathrm{T} \beta 4$. (B) T $\beta 4$ decreased senescence of EPCs in a dosedependent manner. Data are presented as the mean \pm SEM of 3 independent experiments. ${ }^{*} \mathrm{P}<0.05$ compared with control. $\mathrm{T} \beta 4$, thymosin $\beta 4$; EPC, endothelial progenitor cell; SA- $\beta$-gal, senescence-associated $\beta$-galactosidase.

Western blot analysis. Following 7 days of culture, EPCs were deprived of serum for $12 \mathrm{~h}$ to render the cells quiescent. Cells were cultured for an additional $24 \mathrm{~h}$ in the absence or presence of T $\beta 4(1,000 \mathrm{ng} / \mathrm{ml})$ and the PI3K-inhibitor wortmannin or the eNOS inhibitor L-nitroarginine methyl ester hydrochloride (L-NAME; Sigma-Aldrich, St. Louis, MO, USA). EPCs were collected and lysed with lysis buffer as previously described (19). Proteins (50 $\mu \mathrm{g} /$ lane) were separated on SDS-polyacrylamide gels and blotted onto PVDF membranes (Bio-Rad, Hercules, CA, USA). Western blot analysis was performed using anti-

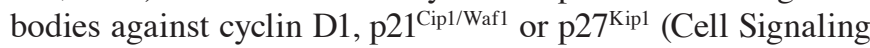
Technology Inc.). Following reaction with enhanced chemiluminescence reagent (Amersham Pharmacia Biotech, Haemek, Israel), images were captured using an image reader LAS 4000 system (Fujifilm, Tokyo, Japan).

Statistical analysis. All experiments were performed at least three times. Data were analyzed by unpaired Student's t-test for comparisons between two groups or one-way ANOVA for multiple comparisons and are expressed as the mean \pm SEM. $\mathrm{P}<0.05$ was considered to indicate a statistically significant difference.

\section{Results}

$T \beta 4$ prevents senescence of EPCs in a dose-dependent manner. EPCs were exposed to T $\beta 4$ at various concentrations $(0,1,10,100$ and $1,000 \mathrm{ng} / \mathrm{ml})$ for $24 \mathrm{~h}$ and the senescence levels were measured by SA- $\beta$-gal staining, which is widely used as a biomarker of senescence (15). As demonstrated in Fig. 1, T $\beta 4$ decreased senescence of EPCs in a dose-dependent manner, with a maximal effect at $1,000 \mathrm{ng} / \mathrm{ml}$.

Effects of T $\beta 4$ on hTERT mRNA expression in EPCs. To investigate the effect of $\mathrm{T} \beta 4$ on the expression of hTERT mRNA, EPCs were treated with T $\beta 4(1,000 \mathrm{ng} / \mathrm{ml})$ for $24 \mathrm{~h}$ 


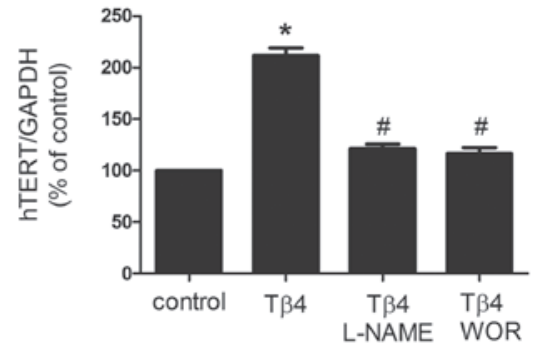

Figure 2. Effect of T $\beta 4$ on hTERT mRNA expression. On day 7, EPCs were treated with $\mathrm{T} \beta 4(1,000 \mathrm{ng} / \mathrm{ml})$ for $24 \mathrm{~h}$ with or without pretreatment with wortmannin $(100 \mathrm{nM})$ or L-NAME $(100 \mu \mathrm{M})$. Data are presented as the mean \pm SEM from a representative experiment performed in triplicate. ${ }^{*} \mathrm{P}<0.05$ vs. control; ${ }^{*} \mathrm{P}<0.05$ vs. cells treated with $\mathrm{T} \beta 4$ only. $\mathrm{T} \beta 4$, thymosin $\beta 4$; hTERT, human telomerase reverse transcriptase; EPCs, endothelial progenitor cells; L-NAME, L-nitroarginine methyl ester hydrochloride.
A

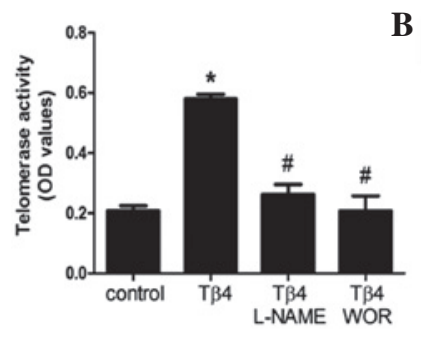

B

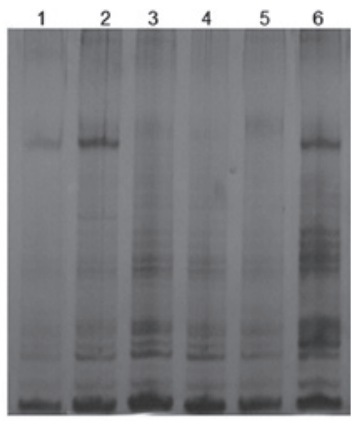

Figure 3. Effect of T $\beta 4$ on telomerase activity. (A) Following 7 days of cultivation, T $\beta 4$ was added for $24 \mathrm{~h}$ with or without pretreatment with wortmannin $(100 \mathrm{nM})$ or L-NAME $(100 \mu \mathrm{M})$ and telomerase activity was measured by the TRAP assay. Data are presented as the means \pm SEM. ${ }^{*} \mathrm{P}<0.05$ compared with control; ${ }^{\#} \mathrm{P}<0.05$ compared with $\mathrm{T} \beta 4$ (1,000 ng/ml). (B) Representative PAGE gel of TRAP-PCR. Lane 1, negative control; lanes 2 and 3, telomerase activity in the presence of $\mathrm{T} \beta 4$ for $24 \mathrm{~h}(0$ and $1,000 \mathrm{ng} / \mathrm{ml})$; lanes 4 and 5 , telomerase activity in the presence of $1,000 \mathrm{ng} / \mathrm{ml} \mathrm{T} \beta 4$ for $24 \mathrm{~h}$ with pretreatment with wortmannin $(100 \mathrm{nM})$ or L-NAME $(100 \mu \mathrm{M})$; lane 6 , positive control. All experiments were repeated at least 3 times and a representative result is presented. T $\beta 4$, thymosin $\beta 4$; L-NAME, L-nitroarginine methyl ester hydrochloride; TRAP, telomeric repeat amplification protocol.

and real-time RT-PCR analysis was performed. As demonstrated in Fig. 2, expression of hTERT mRNA was identified as significantly increased by cotreatment with $\mathrm{T} \beta 4$ for $24 \mathrm{~h}$. Previously, T $\beta 4$ treatment of EPCs was revealed to stimulate time-dependent phosphorylation of Akt and eNOS (8), which is known to regulate the senescence of mature endothelial cells. In the present study, the PI3K inhibitor wortmannin and eNOS inhibitor L-NAME were utilized to assess involvement of the PI3K pathway and eNOS. As revealed in Fig. 2, pretreatment with wortmannin $(100 \mathrm{nM})$ or L-NAME $(100 \mu \mathrm{M})$ for 30 min was identified to significantly reverse the increase in expression of hTERT mRNA induced by T $\beta 4$, suggesting that $\mathrm{T} \beta 4$ affects EPC senescence via the PI3K-Akt-eNOS signal transduction pathway $(\mathrm{P}<0.05)$.

TR4 stimulates telomerase activity in EPCs. Senescence is hypothesized to be triggered by shortening of telomere length (18). Telomere shortening during cell division is counteracted by telomerase activity, therefore, we analyzed telomerase activity in EPCs. As demonstrated in Fig. 3, incubation of EPCs with $\mathrm{T} \beta 4$ was identified to result in a significant increase
$\mathbf{A}$
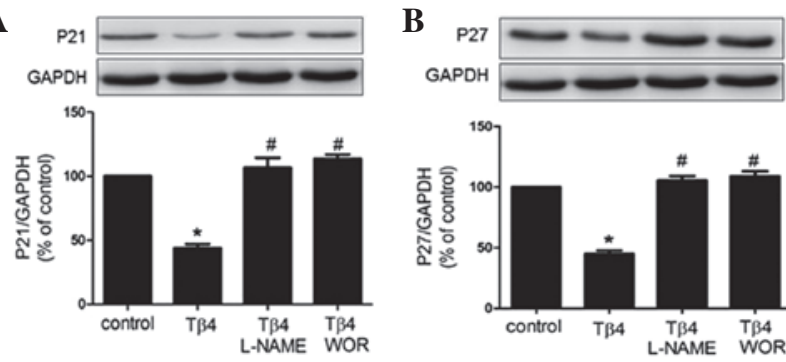

C

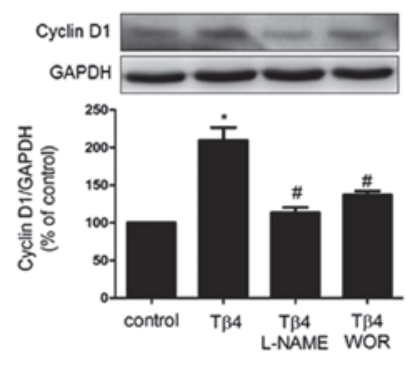

Figure 4. Effect of T $\beta 4$ on expression of p21, p27 and cyclin D1. EPCs on day 7 were treated with $1,000 \mathrm{ng} / \mathrm{ml} \mathrm{T} \beta 4$ with or without pretreatment with wortmannin $(100 \mathrm{nM})$ or L-NAME $(100 \mu \mathrm{M})$ for $30 \mathrm{~min}$. T $\beta 4$-induced EPC senescence was attenuated by wortmannin or L-NAME. Expression of (A) p21, (B) p27 and (C) cyclin D1 was analyzed by western blot analysis. GAPDH served as a loading control. Data are presented as mean \pm SEM $(\mathrm{n}=3)$. ${ }^{*} \mathrm{P}<0.05$ vs. control; ${ }^{\#} \mathrm{P}<0.05$ vs. cells treated with $\mathrm{T} \beta 4$ only. $\mathrm{T} \beta 4$, thymosin $\beta 4$; L-NAME, L-nitroarginine methyl ester hydrochloride; EPCs, endothelial progenitor cells.

in telomerase activity $(\mathrm{P}<0.05)$. The $\mathrm{T} \beta 4$-induced increase in telomerase activity was suppressed by wortmannin $(100 \mathrm{nM})$ or L-NAME $(100 \mu \mathrm{M})$, similar to the effect of these inhibitors on hTERT mRNA expression.

Effects of T $\beta 4$ on cell senescence-related proteins in EPCs. The senescent phenotype is induced by expression of CDKIs. To this end, expression patterns of p21, p27 and cyclin D1 have previously been studied as biomarkers of senescence (12). Therefore, investigation of the expression of the pro-senescent proteins p21 and p27 and the anti-senescent protein cyclin D1 by western blot analysis was performed in the present study. As revealed in Fig. 4, p21 and p27 protein expression was decreased by treatment with $\mathrm{T} \beta 4$ for $24 \mathrm{~h}$, whereas cyclin D1 protein expression was increased. These effects were eliminated by treatment with wortmannin $(100 \mathrm{nM})$ or L-NAME $(100 \mu \mathrm{M})$, suggesting that the PI3K-Akt-eNOS signal transduction pathway is involved in the process by which $T \beta 4$ attenuates EPC senescence.

\section{Discussion}

Results of the present study revealed that exogenous $T \beta 4$ protected EPCs from senescence in a concentration-dependent manner and suggested that this may be associated with activation of telomerase and elongation of telomeres. In addition, the importance of PI3K-Akt-eNOS activation in this process was demonstrated.

The essential role of vascular endothelium in cardiovascular disorders is increasingly being recognized. Although mature endothelial cells contribute to the repair of endothelial 
injury, the cells possess limited regenerative capacities (20). Previous studies indicate that postnatal neovascularization does not rely exclusively on sprouting of mature endothelial cells in pre-existing vessels (angiogenesis) but also involves circulating EPCs $(1,2)$. Importantly, injection of cultivated EPCs enhances neovascularization (21) and this process was identified to improve cardiac function (22). These beneficial properties of EPCs make them attractive candidates for cell therapy that targets the endothelial regeneration of ischemic tissue. However, ex vivo cultivation of EPCs leads to rapid onset of EPC senescence (15), a major risk factor for coronary and peripheral artery disease. In addition, one of the consequences of aging is a decline in the ability of the organism to respond to various stresses, including ischemia. Moreover, the number and functional activities of EPCs are diminished by aging (23). Anti-senescence may be a novel therapeutic strategy for vascular aging and therefore the prevention of cellular senescence of EPCs may have important clinical implications, particularly in diseases associated with increased cellular senescence, including atherosclerosis.

Aging leads to an irreversible state of cell cycle arrest known as replicative senescence, which is associated with telomere shortening (11). Telomerase, a RNA-directed DNA polymerase, extends telomeres of eukaryotic chromosomes and delays the development of senescence. A number of previous studies have demonstrated that telomere length and telomerase activity of circulating EPCs are decreased in patients with various risk factors for coronary artery disease $(5,24)$. Similarly, evidence indicates that EPC subpopulation senescence may be mediated partly by the telomerase-dependent telomere length mechanism. There is a good correlation between the expression of hTERT mRNA and the presence of telomerase activity (25). Overexpression of hTERT by adenovirus-mediated gene delivery delays senescence of EPCs (24). In the present study, telomerase activity in EPCs was increased by treatment with $\mathrm{T} \beta 4$ and this activation was accompanied by increased expression of hTERT mRNA. T $\beta 4$ delayed EPC senescence through telomerase activation, which may be associated with T $\beta 4$-induced upregulation of the expression of hTERT. However, replicative senescence is also regulated by a telomere-independent mechanism. In addition, cellular senescence is induced by DNA damage, cellular stress and oncogenic activation (26). Further studies are required to elucidate the mechanisms underlying the inhibitory effects of $\mathrm{T} \beta 4$ on senescence in EPC.

CDKs regulate and coordinate the eukaryotic cell cycle. CDKs are controlled through several processes, including binding of inhibitory Cip subunits (12). For example, p27 ${ }^{\text {Cip2 }}$ binds to the phosphorylated CDK2-cyclin A complex, interacting with the CDK and cyclin (27), whereas p21 ${ }^{\text {Cip1 }}$ inhibits CDK activity with selectivity for G1/S phase CDK-cyclin complexes (28). The majority of anti-proliferative signals lead to induction of CDK inhibitors. Indeed, senescence augments the expression of cell-cycle inhibitory proteins, including $\mathrm{p} 27^{\mathrm{Kip} 1}$ or $\mathrm{p} 21^{\mathrm{Cip} 1 / \mathrm{Waf} 1}(29,30)$. In the present study, T $\beta 4$ treatment upregulated the expression of cyclin D1 and reduced the expression of p27 and p21. These results suggest that the effects of T $\beta 4$ on EPC senescence involve regulation of cyclin D1, p27 and $\mathrm{p} 21$, providing a mechanism for the control of EPC life span by $\mathrm{T} \beta 4$.
Possible signal transduction pathways involved in the effects of T $\beta 4$ on senescence were also investigated. The PI3K-Akt complex is a critical component of the pathway that regulates signaling of multiple biological and pathophysiological processes, including apoptosis, metabolism, cell migration, cell proliferation and cell growth (31). Previous studies demonstrated that T $\beta 4$ activates the PI3K-Akt-eNOS pathway $(7,8)$ which is required to regulate cellular senescence (32). Therefore, we hypothesized that this pathway may regulate $\mathrm{T} \beta 4$-mediated EPC senescence. In the present study, T $\beta 4$-induced prevention of EPC senescence and upregulation of telomerase activity and hTERT mRNA were significantly attenuated by the PI3K inhibitor (wortmannin) and the eNOS inhibitor (L-NAME) indicating that the PI3K-Akt-eNOS signal transduction pathway is involved in the effects of T $\beta 4$ on EPC senescence.

In conclusion, the results of the present study demonstrate that T $\beta 4$ prevents EPC senescence by activation of the PI3K-Akt-eNOS signaling transduction pathway. These observations require further investigation of EPC-based cytotherapy in clinical applications, particularly in diseases associated with increased cellular senescence, including atherosclerosis.

\section{Acknowledgements}

The present study was supported by the Special Major Projects of Zhejiang Province (2010C13207,N20100746) and the Research Fund for the Doctoral Program of Higher Education (J20110122, 20100101120140).

\section{References}

1. Urbich $\mathrm{C}$ and Dimmeler S: Endothelial progenitor cells: characterization and role in vascular biology. Circ Res 95: 343-353, 2004.

2. Asahara T, Murohara T, Sullivan A, et al: Isolation of putative progenitor endothelial cells for angiogenesis. Science 275: 964-967, 1997.

3. Shi Q, Rafii S, Wu MH, et al: Evidence for circulating bone marrow-derived endothelial cells. Blood 92: 362-367, 1998.

4. Assmus B, Schachinger V, Teupe C, et al: Transplantation of progenitor cells and regeneration enhancement in acute myocardial infarction (TOPCARE-AMI). Circulation 106: 3009-3017, 2002.

5. Vasa M, Fichtlscherer S, Aicher A, et al: Number and migratory activity of circulating endothelial progenitor cells inversely correlate with risk factors for coronary artery disease. Circ Res 89: E1-E7, 2001.

6. Hill JM, Zalos G, Halcox JP, et al: Circulating endothelial progenitor cells, vascular function and cardiovascular risk. N Engl J Med 348: 593-600, 2003.

7. Qiu FY, Song XX, Zheng H, Zhao YB and Fu GS: Thymosin beta4 induces endothelial progenitor cell migration via $\mathrm{PI} 3 \mathrm{~K} / \mathrm{Akt} / \mathrm{eNOS}$ signal transduction pathway. J Cardiovasc Pharmacol 53: 209-214, 2009.

8. Zhao Y, Qiu F, Xu S, Yu L and Fu G: Thymosin beta4 activates integrin-linked kinase and decreases endothelial progenitor cells apoptosis under serum deprivation. J Cell Physiol 226: 2798-2806, 2011.

9. Mathon NF and Lloyd AC: Cell senescence and cancer. Nat Rev Cancer 1: 203-213, 2001.

10. Harley CB, Futcher AB and Greider CW: Telomeres shorten during ageing of human fibroblasts. Nature 345: 458-460, 1990.

11. Collins K: Mammalian telomeres and telomerase. Curr Opin Cell Biol 12: 378-383, 2000.

12. Pavletich NP: Mechanisms of cyclin-dependent kinase regulation: structures of $\mathrm{Cdks}$, their cyclin activators and Cip and INK4 inhibitors. J Mol Biol 287: 821-828, 1999.

13. Dimmeler S, Aicher A, Vasa M, et al: HMG-CoA reductase inhibitors (statins) increase endothelial progenitor cells via the PI 3-kinase/Akt pathway. J Clin Invest 108: 391-397, 2001. 
14. Zheng H, Dai T, Zhou B, et al: SDF-1alpha/CXCR4 decreases endothelial progenitor cells apoptosis under serum deprivation by PI3K/Akt/eNOS pathway. Atherosclerosis 201: $36-42,2008$

15. Assmus B, Urbich C, Aicher A, et al: HMG-CoA reductase inhibitors reduce senescence and increase proliferation of endothelial progenitor cells via regulation of cell cycle regulatory genes. Circ Res 92: 1049-1055, 2003.

16. Chirgwin JM, Przybyla AE, MacDonald RJ and Rutter WJ: Isolation of biologically active ribonucleic acid from sources enriched in ribonuclease. Biochemistry 18: 5294-5299, 1979.

17. Fuller RA, Clark J, Kretzner L, et al: Use of microfluidics chips for the detection of human telomerase RNA. Anal Biochem 313: 331-334, 2003

18. Vasa M, Breitschopf K, Zeiher AM and Dimmeler S: Nitric oxide activates telomerase and delays endothelial cell senescence. Circ Res 87: 540-542, 2000.

19. Zheng H, Fu G, Dai T and Huang H: Migration of endothelial progenitor cells mediated by stromal cell-derived factor-1alpha/CXCR4 via PI3K/Akt/eNOS signal transduction pathway. J Cardiovasc Pharmacol 50: 274-280, 2007.

20. Shantsila E, Watson T and Lip GY: Endothelial progenitor cells in cardiovascular disorders. J Am Coll Cardiol 49: 741-752, 2007.

21. Kalka C, Masuda H, Takahashi T, et al: Transplantation of ex vivo expanded endothelial progenitor cells for therapeutic neovascularization. Proc Natl Acad Sci USA 97: 3422-3427, 2000.

22. Kawamoto A, Gwon HC, Iwaguro $\mathrm{H}$, et al: Therapeutic potential of ex vivo expanded endothelial progenitor cells for myocardial ischemia. Circulation 103: 634-637, 2001

23. Groleau J, Dussault S, Turgeon J, Haddad P and Rivard A: Accelerated vascular aging in CuZnSOD-deficient mice: impact on EPC function and reparative neovascularization. PLoS One 6: e23308, 2011
24. Murasawa S, Llevadot J, Silver M, Isner JM, Losordo DW and Asahara T: Constitutive human telomerase reverse transcriptase expression enhances regenerative properties of endothelial progenitor cells. Circulation 106: 1133-1139, 2002.

25. Greider CW: Telomerase activity, cell proliferation and cancer. Proc Natl Acad Sci USA 95: 90-92, 1998.

26. Serrano M and Blasco MA: Putting the stress on senescence. Curr Opin Cell Biol 13: 748-753, 2001.

27. Russo AA, Jeffrey PD, Patten AK, Massague J and Pavletich NP: Crystal structure of the p27Kip1 cyclin-dependent-kinase inhibitor bound to the cyclin A-Cdk2 complex. Nature 382: 325-331, 1996.

28. Harper JW, Elledge SJ, Keyomarsi K, et al: Inhibition of cyclin-dependent kinases by p21. Mol Biol Cell 6: 387-400, 1995.

29. Collado M, Medema RH, Garcia-Cao I, et al: Inhibition of the phosphoinositide 3-kinase pathway induces a senescence-like arrest mediated by p27Kip1. J Biol Chem 275: 21960-21968, 2000.

30. Macip S, Igarashi M, Fang L, et al: Inhibition of p21-mediated ROS accumulation can rescue p21-induced senescence. EMBO J 21: $2180-2188,2002$.

31. Oudit GY and Penninger JM: Cardiac regulation by phosphoinositide 3-kinases and PTEN. Cardiovasc Res 82: 250-260, 2009.

32. Kang SS, Kwon T, Kwon DY and Do SI: Akt protein kinase enhances human telomerase activity through phosphorylation of telomerase reverse transcriptase subunit. J Biol Chem 274 : 13085-13090, 1999. 